\title{
LOS SISTEMAS BIOINSPIRADOS Y SU ENFOQUE EN LA SOLUCIÓN DE NECESIDADES EN LA INGENIERÍA
}

\section{BIO-INSPIRED SYSTEMS AND THEIR APPROACH TO ENGI- NEERING PROBLEM-SOLVING}

\begin{abstract}
This article presents a brief overview on how different strategies have been developed to take advantage of physical resources and so respond to different social demands until finding innovative solutions based on the behavior of biological systems. Such solutions are based on the emulation or dynamic behaviors that are typical of certain biological systems; therefore, efficiency focuses on the evolution achieved by the analyzed organisms.
\end{abstract}

Keywords: Technology, engineering, bio-inspired system, biology.

\section{Resumen}

El presente artículo hace una breve recuento de cómo se han hallado múltiples estrategias para aprovechar los recursos físicos y atender diversas demandas sociales, hasta llegar a soluciones novedosas basadas en el comportamiento de los sistemas biológicos, estos se fundamentan en la emulación de dinámicas o conductas efectuadas por determinados sistemas biológicos, por ende, centran su eficiencia en la evolución alcanzada por dichos organismos.

Palabras clave: Tecnología, ingeniería, sistemas bioinspirados, biología.
Diego F. Rocha.

Ingeniero Electrónico, MSc. en Ciencias de la Información y las Comunicaciones de la Universidad Distrital "Francisco José de Caldas",

dfrochaa@correo.udistrital.edu. co

Danilo Alfonso López Sarmiento Ingeniero Electrónico, MSc. en Teleinformática, Docente planta de la Universidad Distrital "Francisco José de Caldas", Coordinador de la Maestría en Ciencias de la Información y las Comunicaciones

dalopez@udistrital.edu.co

\section{Ernesto Gómez Vargas}

Ingeniero Electrónico, MSc. en Teleinformática, Candidato a Doctor en Ingenieria, Docente de planta de la Universidad Distrital "Francisco José de Calda" egomez@udistrital.edu.co

Tipo: Artículo de revisión

Fecha de Recepción: Sept. 16 de 2010

Fecha de Aceptación: Nov. 5 de 2010

\section{INTRODUCCIÓN}

La humanidad siempre ha buscado satisfacer sus necesidades por medio del desarrollo de diferentes tecnologías. En un inicio, dichas tecnologías eran bastante simples y de poca elaboración; su implementación se basaba en conocimientos empíricos provenientes de la experiencia. A medida que la sociedad ha ido evolucionando también lo han hecho sus propias necesidades y motivaciones volviéndose más complejas y estructuradas y como es natural, también lo han hecho los métodos y tecnologías por 
medio de las cuales se logra satisfacerlas. En ese sentido, se han producido importantes descubrimientos y planteado multiplicidad de teorías con el fin de brindarle mayor eficiencia a los métodos y tecnologías creadas. De la extensa cantidad de esquemas que permitieron entender mejor las leyes de la naturaleza surgió la ingeniería.

Gracias a esta rama de la ciencia y a sus aplicaciones en numerosas áreas, las sociedades contemporáneas han asistido al advenimiento de prominentes avances que han mejorado su calidad de vida. Obviamente, como también sucede con las personas, los adelantos científicos poseen precedentes importantes, sucesos que ilustran el lugar protagónico ocupado por el que hacer ingenieril durante la historia.

Lejos se está de los años 1643 y 1644, cuando Torricelli comprobó que la presión de la atmósfera es igual a la ejercida por una columna de mercurio de, aproximadamente 76 centímetros de altura [1]. Un experimento posterior evidenció otra situación: al crearse un vacío parcial bajo un émbolo de grandes dimensiones introducido en un cilindro, la fuerza sumada de 50 hombres no resultaría suficiente para evitar la caída del émbolo al fondo del tambor a causa de la presión atmosférica. Tales experimentos dieron vida a una original idea: si se encontrara algún mecanismo sencillo para crear el vacío en repetidas ocasiones, sería factible convertir la presión atmosférica en una ventajosa fuente de energía [2]. En este camino de búsqueda, Denis Papín describió, en 1687, el funcionamiento básico de su máquina neumática: en un cilindro vertical se movía un pistón por efecto del vapor procedente del agua calentada en el fondo del tubo. Con base en los incesantes hallazgos, se articularon los principios para la ulterior creación de la máquina de vapor, y con este elemento, se dio inicio a la Revolución Industrial, fenómeno transformador de todos los ámbitos sociales.

Un proceso similar fue el desarrollado por
Maxwell para formular, en 1873, un modelo matemático que relacionaba la electricidad con el magnetismo. En el marco de sus consideraciones, señaló la existencia de un tipo especial de ondas que al estar formadas por un campo eléctrico y otro magnético, deberían ser llamadas 'electromagnéticas'. En sus indagaciones, el científico constató que la luz era una onda de esta clase. Asimismo, cabe mencionar a Heinrich Hertz, quien en 1887 acopló el primer transmisor de radio, con la capacidad de generar radiofrecuencias comprendidas entre $31 \mathrm{MHz}$ y 1.25 GHZ [3].

La humanidad ha ido hallando múltiples estrategias para aprovechar los recursos físicos y atender diversas demandas sociales. A su vez, motiva una pregunta: ¿y si no sólo se explota el potencial brindado por los recursos naturales, sino que también se logra imitar el comportamiento de los sistemas biológicos? Precisamente, del intento por responder este interrogante, nacieron los Sistemas Bioinspirados. Éstos se fundamentan en la emulación de dinámicas o conductas efectuadas por determinados sistemas biológicos, por ende, centran su eficiencia en la evolución alcanzada por dichos organismos.

\section{ACERCAMIENTO A LOS SISTEMAS BIOINSPIRADOS}

Diferentes algoritmos, inspirados en esos sistemas, han mostrado su aplicabilidad en el planteamiento de alternativas que intentan resolver exigencias contemporáneas, las cuales difícilmente pueden solucionarse por medio de los típicos modelos basados en esquemas matemáticos o físicos. Esta complicación se presenta en la medida que varias conformaciones albergan un alto grado de incertidumbre debido a su poca linealidad. No obstante, las teorías bioinspiradas permiten despejar esas barreras.

En el contexto de los Sistemas Bioinspirados se encuentran los algoritmos ACO (Ant Colony Optimization), cuya operatividad se 
cimienta en el desenvolvimiento de ciertas especies de hormigas: estos insectos, al buscar comida, adoptan un modelo que puede ser llamado "de auto-organización" [4]. Efectivamente, aunque cada individuo actúe con autonomía, sin un ente coordinador de la labor colectiva, el resultado es un orden visible en todo el conjunto, de modo que el desempeño grupal deviene de la interacción entre las unidades. Esta regulación le otorga a la comunidad la posibilidad de adaptarse a los cambios en el ambiente con una gran versatilidad, al punto que la capacita para sobreponerse a las fallas individuales de sus miembros [4].

Desde la óptica de una colonia de hormigas, la auto-organización se da mediante interacciones simples, como son la realimentación positiva (denominada Estigmergia) y el comportamiento aleatorio. La primera da cuenta de un procedimiento de estos insectos, consistente en depositar rastros de feromonas cuando regresan a su hormiguero desde una fuente de comida, con el fin de que esas trazas sean olfateadas y seguidas por los integrantes de la colonia. Por su parte, el segundo indica que cada sujeto, aunque parezca caminar de manera aleatoria, en realidad sigue un recorrido probabilísticamente influenciado por las huellas de feromonas dejadas por sus congéneres. Estos mecanismos son los que un algoritmo ACO intenta emular para resolver problemas de notoria complejidad [4]. De hecho, en razón de su adaptabilidad, este método resulta muy adecuado en el enrutamiento de redes de telecomunicaciones, en particular si se trata de encontrar rutas que varíen con dinamismo y en consonancia con los cambios temporales del tráfico, ocurridos a distintas escalas de tiempo [4].

Otro estudio enfocado en sistemas bioinspirados es el modelo de John Doyle, aplicado en el transporte de paquetes TCP/IP. Éste se sustenta en la técnica empleada por colonias de bacterias Escherichia Coli (cepas productoras de toxina Shiga STEC constituyen un grupo importante dentro del con- junto de patógenos emergentes trasmitidos por alimentos [5]) para transmitir información. Según Doyle, los dispositivos que hoy en día usan la Internet, detectan la congestión en el canal al establecer cuántos de los paquetes enviados se pierden. En concepto del investigador, esto equivale a tratar de conducir por una carretera con sólo mirar lo que aparece seis metros por delante, y de una forma tal, que se incurra en aceleraciones constantes y frenadas intempestivas ante los obstáculos.

El trabajo de Doyle y sus colaboradores ha posibilitado que los dispositivos incorporen más información sobre el flujo de tráfico (a semejanza de lo hecho por la colonia de bacterias) al tener en cuenta el tiempo utilizado por los paquetes para llegar a su destino. Gracias a estos reportes, los dispositivos toman decisiones mucho más inteligentes, condición que redunda en una ventajosa optimización de la velocidad de transmisión: en 2006, este grupo de investigadores envió 17 Gbps a través de Internet [6].

Una muestra más de un Sistema Bioinspirado es la sincronización espontánea, expresada en una infinidad de procesos latentes en el Universo, los cuales involucran desde partículas y átomos hasta planetas y galaxias. Su análisis ha sido compartido por biólogos, físicos, matemáticos, astrónomos, ingenieros, sociólogos y geólogos, entre otros profesionales. Cuatro ejemplos pueden ilustrarla mejor. El corazón, órgano compuesto por cientos de miles de células musculares que para contraerse y relajarse a la par, necesitan los impulsos eléctricos originados por una pequeña región de células. Los espermatozoides, que al dirigirse hacia el óvulo, mueven su cola al unísono en una suerte de nado sincronizado. El rayo láser, cuya coherencia proviene de trillones de átomos pulsantes, emisores ininterrumpidos de fotones dotados de la misma fase y frecuencia. Y las luciérnagas, reconocidas en muchas regiones del mundo por su espectáculo de fulgores brillantes 
y simultáneos que se extiende por kilómetros a lo largo de las márgenes de los ríos. Sobre este último caso, en pruebas de laboratorio, se ha notado una peculiaridad: el destello de estos singulares seres depende del momento de su ciclo de vida por el que atraviesen al recibir el estímulo. Incluso, al tener la facultad de avanzar o atrasar su ciclo, es posible afirmar que disponen de un oscilador interno reiniciable [7].

La sincronización espontánea de las luciérnagas ha servido de plataforma para el desarrollo de investigaciones orientadas a solventar los inconvenientes de las redes ad-hoc. Éstas, debido a sus limitaciones (carecer de infraestructura, recurrir a la transmisión inalámbrica, sufrir cambios topológicos constantes y estar integradas por dispositivos con recursos limitados [7]), registran problemas de coordinación cuando se incrementa el número de sus unidades componentes.

Otra de las técnicas que se reconocen como bioinspiradas, es el proceso de búsqueda estocástico basado en la teoría de selección natural. Esta teoría se resume en la "supervivencia del más apto" de ese modo, a medida que transcurran las generaciones en una población de individuos, los genes que determinen características ventajosas en los organismos que lo posean tenderán a prevalecer sobre los otros. Aquellos descendientes que por mutaciones o entrecruzamiento genético permitan obtener una nueva característica beneficiosa tendrán mayores posibilidades de transferir sus "buenos genes" a las siguientes generaciones pues son quienes potencialmente tendrán mayor cantidad de descendientes. Los algoritmos evolutivos recrean la teoría darwiniana para resolver problemas de búsqueda y optimización. Trabajan con una población de potenciales soluciones al problema a resolver representadas en una estructura de datos que recibe el nombre de cromosoma. A cada solución se le asigna un valor de aptitud, también conocido como fitness, que cuantifica qué tan bien resuelve el problema en cuestión [8].

Cuanto mayor sea este valor mayor será la probabilidad de que la misma sea seleccionada para reproducirse, cruzando su material genético (cromosoma) con el de otra solución. Este cruce producirá nuevas soluciones, descendientes de las anteriores, las cuales comparten algunas de las características de sus padres. Cuanto menor sea el fitness de la solución, menor será la probabilidad de elección para la reproducción, y por tanto tendrá menor oportunidad de que su material genético se propague en sucesivas generaciones. Así, a lo largo de las generaciones, las buenas características se propagan a través de la población, favoreciendo el cruce de los individuos mejor adaptados, siendo exploradas las áreas más prometedoras del espacio de búsqueda. Si el Algoritmo Evolutivo ha sido bien diseñado, la población convergerá, idealmente, hacia una solución óptima del problema [8].

Por su parte, los problemas de diseño de topologías de redes sujeto al cumplimiento de determinadas restricciones tales como minimizar costos, garantizar conectividad, minimizar retardos etc. están comprendidos en una clase de problemas más general conocida con el nombre de optimización combinatoria. Estos últimos pertenecen al conjunto de problemas NP considerados por la comunidad científica como intratables con el modelo de computadora vigente en la actualidad [8].

Con base en lo anterior, el problema del diseño de redes óptimas basado en restricciones puede ser abordarlo utilizando alguna estrategia evolutiva. Se puede modelar una red de información por medio de un grafo formado por nodos y enlaces. A cada enlace se le asocia algún valor que representa el costo o peso de esa conexión. En esencia se trata de algún parámetro que caracteriza el enlace y que interviene directa o indirectamente en el criterio de optimización. En algunos casos se pueden 
considerar simultáneamente más de un parámetro lo que convierte el problema en una optimización multiobjetivo. La utilización de estrategias evolutivas aplicadas en este contexto dan lugar a lo que se conoce como Optimización Evolutiva Multiobjetivo [8].

El problema de hallar el mejor diseño de la red se reduce entonces a encontrar el mejor grafo que garantice las restricciones impuestas. Para resolverlo con alguna estrategia evolutiva es necesario crear la población de cromosomas que representen las potenciales soluciones buscadas. Para ello se debe concebir alguna forma de representar un grafo en un cromosoma [8].

Una vez se establecía la forma de codificar los grafos como cromosomas se está en condiciones de utilizar alguna estrategia evolutiva conocida para resolver el problema de optimización. En ocasiones es posible ensayar alguna variante intentando aprovechar el conocimiento de alguna particularidad del problema en cuestión [8].

En [9] se realiza una investigación sobre el Diseño Optimo de Redes de mínimo costo, sujeto a restricciones de confiabilidad con el supuesto de fallas en los enlaces y propone como estrategia de resolución un algoritmo evolutivo con operadores genéticos ad-hoc enriquecidos con conocimiento específico del problema, lo cual permite acotar el espacio de búsqueda del algoritmo. En la actualidad existe dentro de la comunidad de inteligencia artificial una línea de investigación sobre las estrategias para introducir información específica del problema a resolver en los Algoritmos Evolutivos [8].

En [10] se implementó un Algoritmo Evolutivo para resolver el problema del diseño de redes confiables sobre un enorme espacio de búsqueda obteniendo unos resultados bastante buenos [8].

En [11] se presenta otro método evolutivo para resolver el ruteo multicast con restricción de mínimo costo. Los autores proponen una nueva forma de codificación cromosómica acompañada de una función de reparación que corrige los genotipos inválidos. A diferencia del enfoque convencional que representa dentro del cromosoma una potencial solución al problema -árbol de distribución multicast- esta propuesta busca codificar un grafo genérico [8].

En [12] se presenta una solución evolutiva considerando dos restricciones de calidad de servicio que la red debe proveer: retraso máximo extremo a extremo (end-to-end) y variación máxima del retraso de la transmisión por los distintos caminos desde la fuente a cada uno de los destinos [8].

Generando otro enfoque a los modelos inspirados en los sistemas biológicos, se encuentran las Redes Neuronales Artificiales la cuales emulan a las biológicas, basados en la eficiencia de los procesos llevados a cabo por el cerebro, e inspirados en su funcionamiento, se han utilizado para aprender estrategias de solución basadas en ejemplos típicos de patrones [8].

Los computadores actuales han demostrado ser capaces de resolver cálculos matemáticos complejos a enormes velocidades, no obstante muestran muchas dificultades en la realización de tareas sencillas para los seres humanos, como por ejemplo la identificación de un rostro conocido entre muchos otros [8].

El cerebro humano es capaz de interpretar información imprecisa suministrada por los sentidos a un ritmo extremadamente veloz. Su desempeño, altamente paralelo, no se ve afectado por la pérdida diaria de neuronas. Pero sin duda, su característica principal radica en su capacidad de aprender sin instrucciones explícitas de ninguna clase. A diferencia de los sistemas de cómputo tradicionales, las redes neuronales artificiales no ejecutan instrucciones sino que responden en paralelo a las entradas que 
se les presentan. El conocimiento de una red neuronal no se almacena en instrucciones, sino que se representa a través de su topología y de los valores de las conexiones -pesos- entre neuronas. A diferencia de los sistemas basados en conocimiento -simbólico- deductivos-, las redes neuronales artificiales no basan su funcionamiento en la ejecución de reglas definidas a priori a través de un programa sino que son capaces de extraer sus propias reglas a partir de los ejemplos de aprendizaje. Esto es lo que les permite modificar su comportamiento en función de la experiencia. Una vez que el conocimiento ha sido adquirido, se le pueden presentar a la red datos distintos a los usados durante el aprendizaje. La respuesta obtenida dependerá del parecido de los datos con los ejemplos de entrenamiento. Esto representa la importantísima capacidad de generalización [8].

En el contexto de las redes de datos, es de gran importancia generar mecanismos de predicción de fallos en la red, de manera que se pueda garantizar fiabilidad y calidad de servicio (QoS) en tiempo real para mantener la disponibilidad y fiabilidad de la red e iniciar apropiadas acciones de restauración de la "normalidad". Es por esto que surge la necesidad de implementar sistemas que por medio de análisis del tráfico de la red puedan predecir los fallos en servidores de archivos que se pudiesen presentar tales como time-out y rechazo de conexiones, en [13] se presenta un sistema de predicción de fallos desarrollado con base en redes neuronales artificiales, a las cuáles se les determina de forma experimental y no teórica la arquitectura y los algoritmos de aprendizaje con los que se entrena la red neuronal.

Por otro lado existen los Sistemas Inmunes Artificiales, los cuales están inspirados en el sistema inmune natural. Este es el encargado de la defensa del organismo; controla lo que se introduce en él y lo identifica como propio o no propio. Son varias las características del sistema inmune que resultan de interés para muchos modelos computacionales: capacidad de reconocer cuerpos extraños y anomalías, detección distribuida y tolerancia al ruido -no es necesario una detección exacta de los cuerpos extraños-, y además es capaz de memorizar y reforzar conocimiento sobre los organismos infecciosos -agentes patógenosaprendidos [8].

El sistema inmune está formado por moléculas, células y órganos que están en continua vigilancia de los organismos infecciosos. Cuando un nuevo agente patógeno entra al organismo, el sistema inmune lo detecta, inmoviliza y destruye, pero además lo "memoriza" para poder neutralizarlo más eficientemente la próxima vez que deba enfrentarse con él. Hay dos formas en las que el cuerpo identifica material extraño: el sistema inmune innato y el sistema inmune adquirido. La respuesta inmune innata está basada en la selección negativa, y la adquirida en la selección clonal. Ambas selecciones son importantes en los modelos artificiales de sistemas inmunes. La selección negativa interviene en la obtención de las células llamadas linfocitos $\mathrm{T}$ que son las responsables de identificar lo propio de lo ajeno. Estas células se desarrollan mediante un proceso de variación genética pseudo-aleatoria y luego son sometidas a un proceso de selección negativa dónde son destruidas todas aquellas que reaccionan contra substancias propias del organismo. Obsérvese que este proceso no necesita información alguna sobre ningún agente patógeno [8].

La idea de utilizar principios inmunológicos en la seguridad informática se inició en 1994. Forrest y su grupo de la universidad de Nuevo México trabajaron desde entonces en la creación de un sistema inmune artificial [14]. En 1996 este grupo realizó su primer experimento de detección de intrusiones a partir de llamadas al sistema de procesos UNIX [15]. El sistema recoge información de secuencias de comandos del agente de correo sendmail de UNIX, y la 
utiliza en el período de entrenamiento para definir lo que es propio [8].

Los sistemas inmunes artificiales han demostrado ser útiles y adecuarse bien a las necesidades en el área de seguridad informática. Constituyen una de las técnicas más recientes incorporadas a la construcción de sistemas de detección de intrusos y seguramente continuarán siendo motivo de estudio e investigación [8].

\section{CONCLUSIONES}

Así pues, la evolución del raciocinio humano no deja de sorprender. Al revisar cualquier retrospectiva de las innovaciones diseñadas para satisfacer las necesidades del hombre, causa admiración el paso realizado: de la creación de modelos gestados a partir de la manipulación de fenómenos físicos tal como un día lo hicieron Torricelli, Von Guericke, Papín y Maxwell, entre mu-

Referencias Bibliográficas

[1] J. W. Stewart y M. T. Toral, El mundo de la alta presión, vol. 17. Reverte, 1971.

[2] La máquina de vapor. [Online]. Available: http://thales.cica.es/rd/Recursos/rd99/ed99-0314-01/la_maqva. htm. [Accessed: 22-Jun-2011].

[3] R. Blake, Sistemas electrónicos de comunicaciones. Cengage Learning Latin America, 2004.

[4] M. Alzate y S. Suarez, Dispersión de Tráfico Fractal Mediante Algoritmos Inspirados en Colonias de Hormigas, IEEE Andescon, 2006.

[5] G. Varela et al., «Detection and characterization of Shiga toxin-producing Escherichia coli from clinical cases and food in Uruguay», Revista argentina de microbiología, vol. 40, págs. 93-100, 2008.

[6] This Man Wants To Control the Internet $\mid$ Computers | DISCOVER Ma- chos otros, se ha arribado a la emulación de conductas biológicas que son moldeadas con la intención de obtener de ellas el mayor beneficio.

Este enfoque se caracteriza por crear sistemas que tienen la capacidad de aprender. Lo anterior puede ser obtenido a nivel de individuo imitando el cerebro -Redes Neuronales-, de colonia imitando el comportamiento social de las hormigas -Ant Colony Optimization- o a nivel de especie, imitando la evolución -Computación Evolutiva-.

Estas reproducciones han encontrado un amplio margen de implementación en los sistemas complejos, es decir, aquellos caracterizados por su elevada cantidad de variables, particularidad que les confiere un alto grado de incertidumbre e impide construir sus modelos mediante los típicos algoritmos matemáticos o físicos.

[7] This Man Wants To Control the Internet | Computers | DISCOVER Magazine». [Online]. Available: http:// discovermagazine.com/2007/nov/ this-man-wants-to-control-the-internet. [Accessed: June 23 of 2010].

[8] M. A. Alzate, G. A. Puerta, y E. A. Aguirre, "Effects of topology and mobility in bio-inspired synchronization of mobile ad hoc networks", in Communications (LATINCOM), 2010 IEEE Latin-American Conference on, págs. 1-6.

[9] L. C. Corbalán, «Sistemas inteligentes aplicados a redes de datos», Carrera de Especialidad en Redes, Universidad Nacional de La Plata-ARGENTINA, 2006. 
[10] H. E. Magnago, «Algoritmos evolutivos aplicados a problemas de diseño de redes confiables», Tesis presentada para obtener el grado de Magíster en Redes de Datos, Universidad Nacional de La Plata-ARGENTINA, 2006.

[11] B. Dengiz, F. Altiparmak, y A. E. Smith, «Efficient optimization of allterminal reliable networks, using an evolutionary approach»,, Reliability, IEEE Transactions on, vol. 46, no. 1, págs. 18-26, 1997.

[12] M. Karabi, M. Fathy, y M. Defighan, "QoS multicast routing based on a heuristic genetic algorithm», in Electrical and Computer Engineering, 2004. Canadian Conference on, 2004, vol. 3, págs. 1727-1730 Vol. 3.

[13] M. Hamdan y M. E. El-Hawary, «Multicast routing with delay and delay variation constraints using genetic algorithm», in Electrical and Computer Engineering, 2004. Canadian Conference on, 2004, vol. 4, págs. 23632366 Vol. 4.

[14] G. A. García y O. Salcedo, «Predicción de Fallos en Redes IP empleando Redes Neuronales Artificiales»», Polibits, vol. 41, págs. 67-76, 2010.

[15] S. Forrest, A. S. Perelson, L. Allen, y R. Cherukuri, «Self-nonself discrimination in a computer», in Research in Security and Privacy, 1994. Proceedings., 1994 IEEE Computer Society Symposium on, 1994, págs. 202-212.

[16] S. Forrest, S. A. Hofmeyr, A. Somayaji, y T. A. Longstaff, «A sense of self for unix processes», in Security and Privacy, 1996. Proceedings., 1996 IEEE Symposium on, 1996, págs. 120-128. 